\title{
Robust Wireless Video Multicast using Distributed Antennas-based IEEE 802.11n Infrastructure
}

\author{
Jean Marie Vella ${ }^{\# 1}$, Saviour Zammit ${ }^{\# 2}$ \\ ${ }^{\#}$ Dept. of Communications and Computer Engineering, University of Malta \\ Msida, Malta \\ 1jvel0009@um.edu.mt \\ 2 saviour.zammit@um.edu.mt
}

\begin{abstract}
Wireless Video Multicast is prone to suffer from frequent packet losses, resulting from the fact that the IEEE 802.11 standard does not use any mechanisms such as Acknowledgements and retransmissions, to combat the errors that are common in the dynamic wireless medium. However this paper shows that the infrastructure can be adapted to reduce the wireless errors, by using spatial diversity and spatial expansion to combat the wireless medium's random nature. This is obtained by adding a number of antennas placed equidistantly at the edge of the coverage area, using the same total transmit power that would be used with one transmit antenna. Moreover, this paper shows that since the IEEE 802.11n, the standard which facilitates the use of multiple antennas, states that the maximum number of antennas at any transceiver is four, two Access Points can be used to transmit the same data packet in orthogonal time slots to transmit good quality H.264 video resulting in all nodes experiencing an average Peak Signal to Noise Ratio greater than 36dB for MCS-5 and MCS-6 for a coverage area having radius of $60 \mathrm{~m}$. Since only two time slots are used, these results are obtained with a $1 / 2$ code rate which is an improvement to the code rate used in current cooperative schemes.

Keywords-wireless video multicast; spatial diversity; spatial expansion; cooperative networks; WLAN
\end{abstract}

\section{INTRODUCTION}

Multicasting is an efficient means of transmitting data to a group of users since it transmits one data packet to a multicast group. Hence, instead of transmitting a unicast packet to each interested receiver multicast transmits only one packet. It is highly beneficial to Wireless Local Area Networks (WLANs) because the wireless medium is inherently a broadcast medium, since any receiver can listen to the data transmitted as long as the transmitter and the receiver use the same channel and multiplexing scheme. In fact the research community has studied ways how to increase security of wireless transmission via association, authentication and encryption.

However, despite the fact that the wireless medium is

The research work disclosed in this publication is funded by the Strategic Educational Pathways Scholarship (Malta). The scholarship is part-financed by the European Union European Social Fund (ESF) under Operational Programme II Cohesion Policy 2007-2013, Empowering People for More Jobs and a Better Quality of Life naturally a broadcast medium, multicast over IEEE 802.11 WLAN suffers from a lack of reliability. The IEEE 802.11's compulsory form of reliability for point to point communication is the use of Acknowledgements (ACKs) [1]. When a source transmits a unicast data packet it expects to receive an ACK within an ACK timeout. If it does not receive an ACK it will retransmit the data given it does not exceed the limit imposed on the retransmission times of the same data packet. However, the problem with multicast data is that there is more than one receiver, and hence who should transmit an ACK, since the source node cannot receive feedback from a multiple number of nodes simultaneously? Therefore the IEEE $802.11 \mathrm{a} / \mathrm{b} / \mathrm{g} / \mathrm{n}$ standard does not allow receivers to ACK multicast data. Hence, when a receiver does not receive a data packet successfully it cannot inform the source that it needs a retransmission.

The importance of adding feedback or retransmission to multicast data transmission in order to increase the reliability was studied by the IEEE 802.11aa task group [2] proposing three different schemes under the term Advanced Groupcast with retries $(\mathrm{GCR})$ :

- Changing multicast data into unicast data i.e. if there are $n$ receivers then $n$ unicast transmissions will follow. This method is called Directed Multicast Service (DMS) [3]. This was further studied by Feng et al. [4] who proposed that the I-frames of video are transmitted using DMS while the remaining frames use the legacy multicast.

- Retransmitting every multicast data packet pro-actively; a technique called GCR Unsolicited Retry [5].

- Gathering feedback by polling some or all of the multicast group members, which feedback to the source a bitmap indicating which multicast data packets were received successfully. This last scheme is referred to as GCR Block ACK [5].

There are other drawbacks that result from the lack of ACKs. Another means of increasing the resilience of transmission is data rate adaptation. The Physical layer (PHY) data rate that is used by the IEEE $802.11 \mathrm{a} / \mathrm{b} / \mathrm{g} / \mathrm{n}$ is adapted according to whether a unicast transmission is deemed successful or not. Hence, for example using Auto-Rate Fallback (ARF) [6] when two sequential transmissions fail to 
be followed by an ACK, the PHY data rate is adapted such that a more robust one, hence a slower PHY data rate, is used. Since current IEEE $802.11 \mathrm{a} / \mathrm{b} / \mathrm{g} / \mathrm{n}$ multicast data transmission does not allow feedback from the receivers in the form of an ACK, data rate adaptation is also eliminated when multicast data is transmitted. Hence, a PHY data rate is chosen and used for all multicast data irrespective of the Packet Error Rate (PER) experienced. Usually, the most robust Modulation and Coding Scheme (MCS) possible is employed; for example MCS-0 which for IEEE $802.11 \mathrm{n}$ is $6.5 \mathrm{Mbps}$ when the Guard Interval is $800 \mathrm{~ns}$. The fact that the most robust MCS is used for the multicast data packet results in another problem called the "Performance Anomaly Problem" [7]. Moreover, the elimination of ACK also results in the removal of contention window adaptation, hence when a multicast data packet collides with a unicast data packet, the unicast source performs back-off, adapting its back-off window according to the contention window, but the multicast source is not even aware of the collision and hence performs neither contention window adaptation nor back-off. Hence as stated by Dujovne and Turletti in [8] multicast transmission is unfair on unicast transmission.

For a thorough survey of the research performed on multicast over wireless access networks, the readers are directed to [9]. The paper is organized in the following manner. First a discussion of how one may deploy a distributed antennas-based infrastructure is presented in Section II. Then the simulation methodology employed to compare three different infrastructures is discussed in Section III. Section IV presents the results obtained highlighting the effect of each infrastructure on the PER and the average PeakSignal to Noise Ratio (PSNR). In Section V, an antenna selection technique is proposed such that the Distributed Antennas-based Infrastructure may also be used for IEEE $802.11 \mathrm{n}$ unicast transmission. The paper is then concluded in Section VI.

\section{Distributed ANTENNAS-BASED INFRASTRUCTURE}

This study forms part of a research initiative which aims to increase the resilience of wireless video multicast by augmenting the infrastructure with special interest to IEEE 802.11 n WLANs. One of the constraints adopted by the study is that changes are performed at the infrastructure end and any modifications at the receivers are performed at the application layer. Since some IEEE $802.11 \mathrm{n}$ receivers have one receive antenna, it is proposed in this paper that wireless multicast over IEEE 802.11n WLAN employs only MCSs which transmit one spatial stream therefore eliminating spatial multiplexing. Also due to single-stream IEEE 802.11n chipsets, Space-Time Block Coding (STBC) is also not recommended in this study. Hence any IEEE 802.11n receiver can join the multicast group and benefit from the enhancements obtained from this study.

Infrastructure-Dependent Wireless Multicast (IDWM), the technique studied in this paper, refers to an infrastructure that is more resilient to packet loss than the legacy infrastructure which places all the transmit antennas of the Access Point (AP) in the middle of the coverage area. This study has already shown both empirically [10], [11] and analytically [12] that by placing antennas at opposite ends of a coverage area, a smaller PER can be experienced. However, the work in [10], [11] and [12] is limited to a two antenna system without application layer redundancy. This is possible because the IEEE 802.11n uses Spatial Expansion when it transmits the data using any one of the MCS-0 to MCS-7 i.e. one spatial stream with more than one transmit antenna. Thus each transmit antenna sends a copy of the data, cyclically shifted with a unique delay to enhance the effect of multipath diversity. This is obtained by using a spatial mapping matrix, $\mathbf{Q}_{k}$ which is defined by (1),

$$
\mathbf{Q}_{k}=\mathbf{M}_{C S D}(k) \bullet \mathbf{D}
$$

where $\mathbf{M}_{C S D}$ is a Cyclic Shift Diversity (CSD) matrix which is a diagonal matrix having elements given by (2).

$$
\left[\mathbf{M}_{C S D}(k)\right]_{i, i}=\exp \left(-j 2 \pi k \Delta_{F} t_{C S}^{i}\right) ; i=1, \ldots, N_{T X}
$$

where $k$ is the index of the subcarrier considered, $\Delta_{F}$ is the subcarrier frequency spacing, $t_{c s}^{i}$ is the CSD for stream transmitted from transmit antenna $i$. The total number of transmit antennas is given by $N_{T X}$. D is a matrix whose size and elements is defined by the IEEE 802.11n [1]; for example, if one space-time stream is to be transmitted using four transmit antennas then $\mathbf{D}$ is given by (3)

$$
\mathbf{D}=\frac{1}{2}\left[\begin{array}{llll}
1 & 1 & 1 & 1
\end{array}\right]^{T}
$$

where []$^{T}$ is the transpose of a matrix.

In this paper, the ideal antenna distribution for a coverage area with a radius of $60 \mathrm{~m}$ is presented. Hence the area considered can model a football ground or a concert arena. Instead of using an IEEE 802.11n AP and placing the transmit antennas at one location with a separation between each antenna, this study proposes that one should take advantage of the fact that the IEEE 802.11n standard includes the use of a maximum of four transmit antennas. Hence an AP such as QHS600 [13] may be used, placing one antenna at the middle of the coverage area and the remaining three placed at equal separation from each other, at the edge of the area of interest as shown in Fig. 1. To deploy this set-up, extension cables

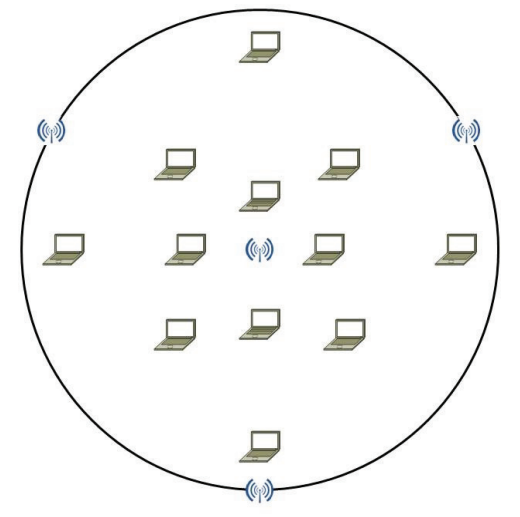

Fig. 1. An Infrastructure using an Access Point with four transmit antennas 


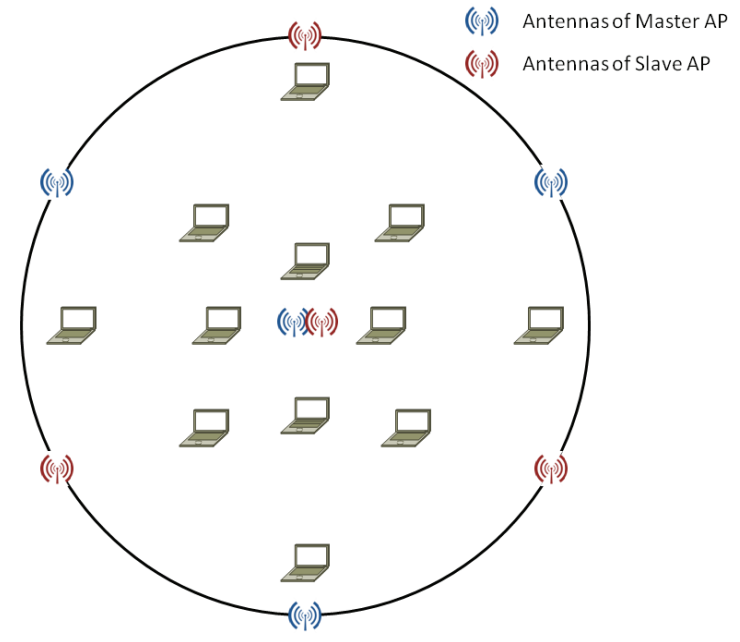

Fig. 2. An Infrastructure using two Access Points each having four transmit antennas

must be used. In order to overcome the loss introduced by the extension cables, antenna amplifiers should be employed to amplify the power to the original transmit power at each antenna.

Distributing the antennas in order to increase the throughput was considered for the next-generation mobile cellular networks by placing the antennas equidistantly in the middle of the radius and not at the edge of the coverage [14]. In this paper, besides proposing a different antenna distribution, a further improvement is proposed,. using two APs each with four transmit antennas and interleaving the antennas as shown in Fig. 2.

The IEEE 802.11 standard does not allow receivers to associate with more than one AP. However as proposed by Miu et al. in [15], if the two APs operate using the same Basic Service Set ID, same Medium Access Control (MAC) address and same channel, the receivers will receive data from both APs since they cannot distinguish between the two. In [16], it was shown how wireless multicast can benefit from the employment of multiple APs using a master-slave relationship i.e. the Master AP takes care of the transmission of all frames including management frames that handle the association and authentication of the receivers and the Slave APs are used to increase resilience of the wireless multicast transmission. However, this could also be used to increase the reliability of transmission from the nodes to APs since in such an uplink the AP is implementing strong receive diversity via spatial diversity. When a Master and Slave AP setup is used, a multicast data packet is first transmitted from the Master AP and then the same packet is transmitted from the Slave AP. Orthogonality between transmissions is achieved using different time slots for transmission from Master and from Slave APs.

Alternatively, the setup of Fig. 2, can also be implemented by using an AP with seven antennas, employing antenna selection on three pairs of these antennas. Hence for the first transmission it uses four of these antennas and for the retransmission of that same packet, the AP employs again the antenna in the middle but switches to the three other previously unused antennas. In such a manner the AP still works according to the specification of the IEEE $802.11 \mathrm{n}$ standard since at one time slot it uses only four antennas to transmit.

\section{Simulation Methodology}

Since the Infrastructures proposed exhibit symmetry, an IEEE 802.11n WLAN sector was simulated consisting of 32 multicast group members placed in a coverage area of radius $60 \mathrm{~m}$, located as shown in Fig. 3 and Fig. 4 in relation to the antennas as depicted in Fig. 2. A worst-case scenario is assumed by simulating receivers that have a single-stream chipset. Three scenarios are considered which are tabulated in Table I.

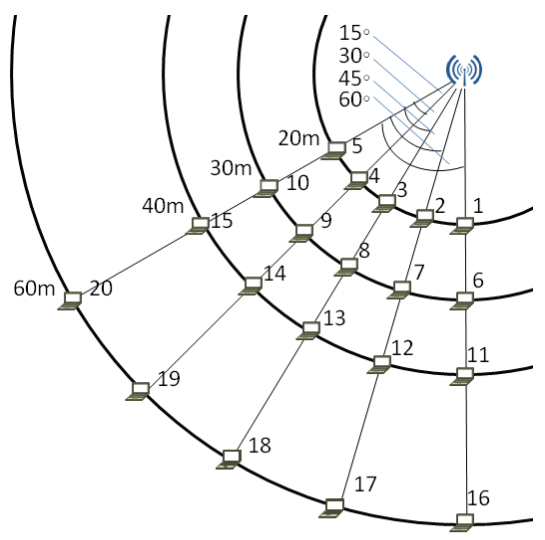

Fig. 3. Distribution of nodes 1 to 20

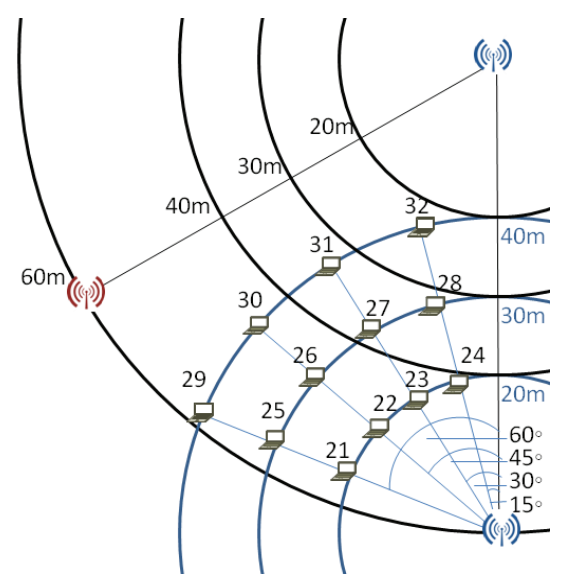

Fig. 4. Distribution of nodes 21 to 32 
TABLE I

The three Infrastructure Setups studies

\begin{tabular}{|c|l|}
\hline \hline Case & \multicolumn{1}{|c|}{ Description } \\
\hline \hline $\boldsymbol{A}$ & $\begin{array}{l}\text { One AP with one transmit antenna located at the middle } \\
\text { of the coverage area. }\end{array}$ \\
\hline $\boldsymbol{B}$ & $\begin{array}{l}\text { One AP with four transmit antennas placed as shown in } \\
\text { Fig. 1 }\end{array}$ \\
\hline $\boldsymbol{C}$ & $\begin{array}{l}\text { Two APs each with four transmit antennas or a single AP } \\
\text { employing antenna selection with the transmit antennas } \\
\text { placed as shown in Fig. 2 }\end{array}$ \\
\hline \hline
\end{tabular}

In order to extract the IEEE 802.11n PHY, the Matlab script created by Schumacher et al. [17] was used. This code assumes a Uniform Linear Array (ULA), hence since the four transmit antennas of the Master AP and of the Slave AP and the single receive antenna of each receiver do not form a ULA, the $4 \times 1$ channel matrix $\mathbf{H}$, between each AP and each receiver was extracted by using the Matlab script to extract first the $1 \times 1$ channel matrix between each antenna and each receiver and then combining them in a $4 \times 1$ matrix. Similarly for Case A i.e. an infrastructure Basic Service Set which consists of undistributed antennas, the channel model was also extracted using this Matlab script. For simplicity, in Case A, it was assumed that the AP had one transmit antenna, thus $1 \times 1$ channel matrices describing the channel between the AP in the middle of the coverage area and the receivers were evaluated. The reason why an AP with one transmit antenna in the middle of the coverage area was used instead of simulating four transmit antennas separated by at least $\lambda / 2 \mathrm{~m}$, where $\lambda$ is the wavelength, is due to the fact that Spatial Expansion alone without Spatial Diversity results in only a theoretical $2 \mathrm{~dB}$ improvement at a PER of $1 \%$ [18].

Since this study focuses on a WLAN deployed in an outdoor environment the channel matrices were extracted using channel model F. However the Matlab script [17] was modified so that all channels simulated a Line-of-Sight (LOS) environment. The IEEE TGn had set the breakpoint of channel model $\mathrm{F}$ at 30m [19], therefore the original Matlab script models a NonLOS (NLOS) environment for channels having a distance of more than $30 \mathrm{~m}$. It was also assumed that a channel remains constant during one packet transmission. For each channel matrix, the Matlab script was used to extract 50,000 channel instances. In order to eliminate the effect of randomness, for each receiver 10 channel matrices consisting of 50,000 channel instances were generated.

The channel matrices were then used by another Matlab script which outputs the simplified Gilbert model parameters $\mathrm{P}_{\mathrm{gb}}$ and $\mathrm{P}_{\mathrm{bg}}$ for every channel matrix. The simplified Gilbert model is a commonly used channel model which receives a packet successfully when it is in the good state but results in an erroneous reception when the state is bad. The channel changes state from good to bad with probability $\mathrm{P}_{\mathrm{gb}}$ and vice versa with probability $\mathrm{P}_{\mathrm{bg}}$.

The simplified Gilbert model parameters were extracted by first using the channel matrices and the tapped delay line model to extract the frequency response of the channel. Then using this frequency response, the post processing SNR for subcarrier $k, \gamma_{k}$ could be evaluated using the equation given by (4) for a Single Input Single Output Channel and the equation given by (5) for the Multiple Input Single Output Channel, where $P_{t x}$ is the total transmit power and $h_{i}$ is the channel gain between transmit antenna $i$ and the single antenna at the receiver. The additive white Gaussian noise has zeromean and variance $\sigma^{2}$. The values of $t_{c s}^{i}$ are specified by the standard [1] and are tabulated in Table II.

$$
\begin{gathered}
\gamma_{k}=\left|\mathbf{H}_{k}\right|^{2} \frac{P_{t x}}{\sigma^{2}} \\
\gamma_{k}=\mid\left(\left.\sum_{i=1}^{4} h_{i} \exp \left(-j 2 \pi k \Delta_{F} t_{c s}^{i}\right)\right|^{2} \frac{P_{t x}}{4 \sigma^{2}}\right.
\end{gathered}
$$

From the post-processing SNR, the effective SNR was evaluated using the method proposed by Bjerk et al. in [20]. Hence for each channel instance, the effective SNR was evaluated. Then the effective SNR was used to calculate the bit error rate (BER) for the MCS considered. Using this BER, the probability of receiving an OFDM symbol correctly was evaluated, which is subsequently used to output the PER. This method of calculating the PER for each channel instance from the effective SNR was similarly used by Xia et al. in [21] and the necessary equations were presented in [12]. Then using a weighted coin toss [20], the Matlab script decides whether a packet is received in error or not. Considering the entire 50,000 packet sequence, the Matlab script outputs $\mathrm{P}_{\mathrm{gb}}$ and $\mathrm{P}_{\mathrm{bg}}$. An average $P_{g b}$ and $P_{b g}$ is then obtained for each 10 channel matrices generated for each location and each case of Table I studied. The simplified Gilbert model parameters were obtained by considering the MCS used, the location of the receiver relative to the antenna/s of the $\mathrm{AP} / \mathrm{s}$ and the $\mathrm{PSDU}$ size. Since video data encoded using H.264 results in different payload sizes, the PSDU sizes considered varied from 100 bytes up to 1000 bytes incremented in steps of 100 bytes.

TABLE II

CYCLIC SHIFT DELAY

\begin{tabular}{|c|l|}
\hline \hline $\mathbf{i}$ & \multicolumn{1}{|c|}{$t_{c s}^{i}$} \\
\hline \hline 1 & $0 \mathrm{~ns}$ \\
\hline 2 & $-400 \mathrm{~ns}$ \\
\hline 3 & $-200 \mathrm{~ns}$ \\
\hline 4 & $-600 \mathrm{~ns}$ \\
\hline \hline
\end{tabular}

TABLE III

PHYSICAL LAYER PARAMETERS

\begin{tabular}{|l|l|}
\hline \hline Carrier Frequency & $5.25 \mathrm{GHz}$ \\
\hline Signal Bandwidth & $20.0 \mathrm{MHz}$ \\
\hline Data Subcarriers & 52 \\
\hline Pilot Subcarriers & 4 \\
\hline Preamble Format & Greenfield Mode \\
\hline Guard Interval & $800 \mathrm{~ns}$ \\
\hline Transmit Power & $20 \mathrm{dBm}$ \\
\hline Noise Figure & $10 \mathrm{~dB}$ \\
\hline Antenna Gain & $0.0 \mathrm{dBi}$ \\
\hline Antenna Pattern & Omni-Directional \\
\hline \hline
\end{tabular}


The Physical layer parameters used to model the channel matrices and extract the simplified Gilbert parameters are tabulated in Table III.

The simplified Gilbert Model parameters are inserted in ns 3.13 [22]. The ns3.13 is also modified to include the IEEE 802.11n MCS-5, 6, 7 and the Greenfield preamble durations. The payload considered is football [23] encoded at $6 \mathrm{Mbps}$ using the H.264 reference software JM18.2 employing the Baseline profile. The football sequence has Common Intermediate Format (CIF) and is encoded at 30 frames per second. Slice mode coding such that the size of each slice does not exceed a size of 926 bytes was employed. Each Group of Pictures consisted of 16 frames, starting with an I-frame and the remaining frames being P-frames. The football sequence was encoded into 7385 packets of differing size. Since the JM18.2 does not decode slice level errors, ffmpeg 0.11 [24] was used for decoding. Due to lost packets some frames could not be decoded, hence the ffmpeg 0.11 was modified in order to output the Group of Picture reference number of those frames that it decodes. Missing frames were then replaced by using the previous frame as error concealment. As tabulated in Table I, three cases were studied. Since Case 3 involves the retransmission of every data packet from the second AP, ns3.13 was used such that for all three cases, every packet transmitted is immediately retransmitted. Since each packet is retransmitted, an application level header was used which states the sequence number of the data packet received. Hence if a packet was already received, it will be discarded otherwise it will be concatenated to the video file. Each case was studied using 25 simulation runs and then obtaining the average PER for each node considered. Moreover, the PSNR was also obtained for each node.

\section{RESULTS}

In this section, the results obtained are presented. First, the PER and the PSNR which each node experiences using the Infrastructure of Case A is compared with the results obtained using the Infrastructure of Case B depicted in Fig. 1. Then the effect of using two APs having the antennas distributed as shown in Fig. 2 is presented, highlighting the improvement that can be attained when spatial diversity is exploited.

\section{A. One AP - Case A vs Case B}

The nodes which benefit the most from the distribution of the transmit antennas as depicted in Fig. 1, are nodes 16, 17, 21, 22, 23 and 24, which as can be noted from Table IV and Table V for MCS 5 and MCS-6 improved the PSNR such that the videos decoded for these two nodes exceeded the minimum PSNR necessary for H.264 i.e. 36dB [25]. These nodes happen to be located close to the antenna placed at the edge of the coverage area as shown in Fig. 1. The node which benefitted the most from the distribution of the four antennas of Fig. 1, with respect to video quality is node 16, which happens to be located exactly underneath the antenna placed at the peripheral of the coverage, assuming omni-directional antennas. But by paying attention to node 23 , one notices that by dispersing the antennas, the PSNR increased by $10.75 \mathrm{~dB}$,
$11.41 \mathrm{~dB}$ and $8 \mathrm{~dB}$ for MCS-5, 6 and 7 respectively. Node 22's PSNR was mitigated by $12.60 \mathrm{~dB}, 12.24 \mathrm{~dB}$ and $6.88 \mathrm{~dB}$ for MCS-5, 6 and 7 respectively. However, for MCS-7 although these six nodes still experienced an improvement in the average PER, only Node 16 had an augmentation in the PSNR enough to result in good quality H.264 i.e. $36 \mathrm{~dB}$.

Node 18, also attained an improvement in PER when the Infrastructure of Case B was used instead of that of Case A, for the three MCS considered. Node 18 enjoyed an increase in average PER by a factor of 15.4, 7.36 and 2.3 for MCS-5, 6 and 7 respectively. Moreover, Nodes 7 and 11 attained a PSNR greater than $36 \mathrm{~dB}$ for MCS-7, whereas with Infrastructure of case A i.e. an AP with one antenna, their PSNR was $32.34 \mathrm{~dB}$ and $29.03 \mathrm{~dB}$ respectively.

For MCS-7, nodes 1, 2, 3, 4 and 5 experienced a considerable increase in PER when the transmit antennas were distributed around the coverage area, enough to reduce the PSNR by an average of $11.32 \mathrm{~dB}$. This is expected because of the decrease in transmit power, which initially was $20 \mathrm{dBm}$ emitted from a distance of $20 \mathrm{~m}$ while the distribution of the antennas results in only $14 \mathrm{dBm}$ emitted from a distance of $20 \mathrm{~m}$. However, the resulting PSNR of these 5 nodes for MCS5 and 6 exceeded $44 \mathrm{~dB}$ with the deployment of Infrastructure

TABLE IV

RESUlTS FOR CASE A AND CASE B FOR MCS-5

\begin{tabular}{|c|c|c|c|c|}
\hline & \multicolumn{2}{|c|}{ Case A } & \multicolumn{2}{|c|}{ Case B } \\
\hline Node & $\begin{array}{c}\text { PER } \\
(\%)\end{array}$ & $\begin{array}{c}\text { PSNR } \\
\text { (dB) }\end{array}$ & $\begin{array}{c}\text { PER } \\
(\%)\end{array}$ & $\begin{array}{c}\text { PSNR } \\
\text { (dB) }\end{array}$ \\
\hline 1 & 0.00 & 47.12 & 0.00 & 46.96 \\
\hline 2 & 0.00 & 47.17 & 0.00 & 47.12 \\
\hline 3 & 0.00 & 47.17 & 0.01 & 46.41 \\
\hline 4 & 0.00 & 47.17 & 0.00 & 47.14 \\
\hline 5 & 0.00 & 47.17 & 0.00 & 46.84 \\
\hline 6 & 0.01 & 46.49 & 0.03 & 45.45 \\
\hline 7 & 0.01 & 46.20 & 0.04 & 44.60 \\
\hline 8 & 0.01 & 46.81 & 0.06 & 43.96 \\
\hline 9 & 0.01 & 46.50 & 0.06 & 44.77 \\
\hline 10 & 0.01 & 46.54 & 0.05 & 45.06 \\
\hline 11 & 0.28 & 39.15 & 0.00 & 47.17 \\
\hline 12 & 0.25 & 39.54 & 0.02 & 45.92 \\
\hline 13 & 0.25 & 39.65 & 0.09 & 42.55 \\
\hline 14 & 0.24 & 39.56 & 0.18 & 40.42 \\
\hline 15 & 0.26 & 39.39 & 0.38 & 38.16 \\
\hline 16 & 6.33 & 26.71 & 0.00 & 47.17 \\
\hline 17 & 6.25 & 26.76 & 0.00 & 47.16 \\
\hline 18 & 6.30 & 26.68 & 0.41 & 37.59 \\
\hline 19 & 6.11 & 26.85 & 1.97 & 31.30 \\
\hline 20 & 6.15 & 26.85 & 3.81 & 28.66 \\
\hline 21 & 3.37 & 29.10 & 0.11 & 41.92 \\
\hline 22 & 1.58 & 32.00 & 0.05 & 44.60 \\
\hline 23 & 0.96 & 34.04 & 0.03 & 44.79 \\
\hline 24 & 0.27 & 39.15 & 0.01 & 47.02 \\
\hline 25 & 3.96 & 28.51 & 0.32 & 38.71 \\
\hline 26 & 1.04 & 33.65 & 0.25 & 39.39 \\
\hline 27 & 0.10 & 42.78 & 0.21 & 40.07 \\
\hline 28 & 0.04 & 44.90 & 0.02 & 46.15 \\
\hline 29 & 4.92 & 27.74 & 1.09 & 33.73 \\
\hline 30 & 0.54 & 36.58 & 0.25 & 39.68 \\
\hline 31 & 0.07 & 44.44 & 0.10 & 42.93 \\
\hline 32 & 0.01 & 46.61 & 0.01 & 46.10 \\
\hline
\end{tabular}


TABLE V

Results For CASE A AND CASE B FOR MCS-6

\begin{tabular}{|c|c|c|c|c|}
\hline & \multicolumn{2}{|c|}{ Case A } & \multicolumn{2}{|c|}{ Case B } \\
\hline Node & $\begin{array}{c}\text { PER } \\
(\%)\end{array}$ & $\begin{array}{c}\text { PSNR } \\
\text { (dB) }\end{array}$ & $\begin{array}{c}\text { PER } \\
(\%)\end{array}$ & $\begin{array}{c}\text { PSNR } \\
\text { (dB) }\end{array}$ \\
\hline 1 & 0.00 & 47.10 & 0.02 & 46.02 \\
\hline 2 & 0.00 & 47.17 & 0.02 & 46.08 \\
\hline 3 & 0.00 & 47.10 & 0.03 & 45.80 \\
\hline 4 & 0.00 & 47.17 & 0.04 & 44.76 \\
\hline 5 & 0.00 & 47.17 & 0.01 & 46.14 \\
\hline 6 & 0.06 & 43.89 & 0.11 & 42.50 \\
\hline 7 & 0.08 & 43.63 & 0.21 & 40.42 \\
\hline 8 & 0.08 & 43.71 & 0.30 & 38.55 \\
\hline 9 & 0.08 & 43.23 & 0.36 & 37.87 \\
\hline 10 & 0.10 & 42.64 & 0.61 & 35.94 \\
\hline 11 & 0.51 & 36.39 & 0.30 & 38.58 \\
\hline 12 & 0.53 & 36.73 & 0.63 & 35.96 \\
\hline 13 & 0.52 & 36.41 & 0.76 & 34.53 \\
\hline 14 & 0.51 & 36.42 & 1.09 & 33.50 \\
\hline 15 & 0.52 & 36.63 & 6.48 & 26.66 \\
\hline 16 & 11.82 & 24.57 & 0.00 & 47.17 \\
\hline 17 & 12.09 & 24.46 & 0.06 & 44.07 \\
\hline 18 & 11.85 & 24.58 & 1.61 & 32.07 \\
\hline 19 & 11.99 & 24.45 & 5.67 & 27.07 \\
\hline 20 & 11.91 & 24.56 & 14.00 & 24.12 \\
\hline 21 & 7.81 & 26.06 & 0.43 & 37.53 \\
\hline 22 & 4.60 & 27.80 & 0.21 & 40.04 \\
\hline 23 & 2.46 & 30.25 & 0.15 & 41.66 \\
\hline 24 & 1.02 & 34.02 & 0.07 & 43.99 \\
\hline 25 & 8.54 & 25.71 & 2.05 & 31.09 \\
\hline 26 & 2.73 & 29.77 & 1.59 & 32.01 \\
\hline 27 & 0.36 & 38.51 & 1.67 & 31.98 \\
\hline 28 & 0.16 & 41.00 & 0.09 & 42.46 \\
\hline 29 & 10.35 & 24.95 & 4.83 & 27.72 \\
\hline 30 & 1.52 & 32.21 & 3.07 & 29.58 \\
\hline 31 & 0.24 & 39.78 & 0.38 & 38.10 \\
\hline 32 & 0.01 & 46.52 & 0.11 & 42.66 \\
\hline
\end{tabular}

B. A similar effect was experienced at node 32. A large increase in PER was observed by nodes 8,9 and 10, in fact considering MCS-7, these three nodes were experiencing an average PER of $1.50 \%$ but this increased to $4.60 \%$ when the four transmit antennas were distributed across the coverage area. Hence for MCS-7 the use of distributing the antennas as depicted in Fig. 1, wasn't beneficial because with one antenna, the nodes close to the middle of the coverage area were experiencing good channel quality. But this was degraded, when the antennas were distributed as shown in Fig. 1, resulting in node 5 achieving a PSNR of $33.19 \mathrm{~dB}$ whereas with an AP having one transmit antenna, it had a PSNR of $46.33 \mathrm{~dB}$. However, a smaller coverage area should be used for the leastresilient MCS, because as expected the higher the MCS considered the smaller the resulting coverage area.

\section{B. Two APs - Case C}

Although in this paper all three case studies use the same code rate i.e. a code rate of $1 / 2$ by immediately retransmitting every packet, it was found that the results obtained using the infrastructure in Fig. 2 outperform the results obtained by the other two cases as can be seen from Table VII. One
TABLE VI

RESUltS FOR CASE A AND CASE B FOR MCS-7

\begin{tabular}{|c|c|c|c|c|}
\hline & \multicolumn{2}{|c|}{ Case A } & \multicolumn{2}{|c|}{ Case B } \\
\hline Node & $\begin{array}{c}\text { PER } \\
(\%)\end{array}$ & $\begin{array}{c}\text { PSNR } \\
\text { (dB) }\end{array}$ & $\begin{array}{l}\text { PER } \\
(\%)\end{array}$ & $\begin{array}{c}\text { PSNR } \\
\text { (dB) }\end{array}$ \\
\hline 1 & 0.01 & 46.21 & 0.88 & 34.28 \\
\hline 2 & 0.01 & 46.18 & 0.63 & 35.93 \\
\hline 3 & 0.02 & 46.09 & 0.66 & 35.49 \\
\hline 4 & 0.01 & 46.16 & 0.51 & 36.94 \\
\hline 5 & 0.01 & 46.33 & 1.19 & 33.19 \\
\hline 6 & 1.49 & 32.28 & 1.34 & 32.76 \\
\hline 7 & 1.50 & 32.34 & 0.64 & 36.05 \\
\hline 8 & 1.44 & 32.44 & 5.42 & 27.41 \\
\hline 9 & 1.55 & 32.05 & 4.67 & 27.79 \\
\hline 10 & 1.47 & 32.43 & 3.61 & 28.90 \\
\hline 11 & 3.57 & 29.03 & 0.58 & 36.67 \\
\hline 12 & 3.38 & 29.12 & 1.51 & 32.17 \\
\hline 13 & 3.48 & 29.14 & 12.32 & 24.50 \\
\hline 14 & 3.52 & 28.97 & 14.35 & 24.01 \\
\hline 15 & 3.46 & 29.00 & 14.53 & 23.95 \\
\hline 16 & 34.48 & 21.51 & 0.00 & 47.17 \\
\hline 17 & 34.76 & 21.55 & 0.91 & 34.54 \\
\hline 18 & 34.71 & 21.54 & 15.10 & 23.86 \\
\hline 19 & 34.97 & 21.56 & 32.76 & 21.79 \\
\hline 20 & 34.61 & 21.57 & 55.62 & 20.64 \\
\hline 21 & 30.92 & 21.80 & 3.91 & 28.48 \\
\hline 22 & 17.25 & 23.42 & 2.56 & 30.30 \\
\hline 23 & 12.93 & 24.28 & 1.47 & 32.28 \\
\hline 24 & 4.76 & 27.75 & 1.36 & 32.76 \\
\hline 25 & 33.63 & 21.59 & 15.58 & 23.78 \\
\hline 26 & 15.56 & 23.68 & 8.35 & 25.75 \\
\hline 27 & 2.87 & 29.69 & 3.37 & 29.08 \\
\hline 28 & 2.16 & 30.65 & 2.56 & 30.06 \\
\hline 29 & 34.12 & 21.62 & 35.49 & 21.58 \\
\hline 30 & 10.53 & 24.94 & 17.91 & 23.33 \\
\hline 31 & 2.41 & 30.47 & 2.88 & 29.60 \\
\hline 32 & 0.17 & 40.72 & 0.78 & 34.99 \\
\hline
\end{tabular}

immediately notices the considerable reduction in PER experienced over all three MCS when 8 antennas were distributed around the coverage area. In fact the maximum PER experienced was that of $0.05 \%, 0.58 \%$ and $6.86 \%$ for MCS-5, MCS-6 and MCS-7 respectively. This contrasts with the maximum PER experienced when MCS-5, MCS- 6 and MCS-7 were employed for an AP with one transmit antenna (Case A), i.e. $6.33 \%, 12.09 \%$ and $34.97 \%$ respectively. The maximum PER obtained when one AP with four transmit antennas deployed as shown in Fig. 1 was used were 3.81\%, $14.00 \%$ and $55.62 \%$

Another salient observation is that with the use of MCS-5 and MCS-6 and the Infrastructure depicted in Fig. 2, the entire multicast group obtained an average PSNR greater than or equal to $36 \mathrm{~dB}$. In fact nodes $1,2,3,4$ and 5 maintained a similar average PSNR as that of Case A i.e. an infrastructure without distributed antennas. These nodes experienced a degradation in PER and in PSNR especially with the use of MCS-7 when the Infrastructure of Case B, the one depicted in Fig. 1, was studied. In fact all nodes achieved an improvement with the setup of Fig. 2, especially node 16 and node 20 which 
TABLE VII

RESUlTS FOR CASE C FOR MCS-5, 6 AND 7

\begin{tabular}{|c|c|c|c|c|c|c|}
\hline \hline & \multicolumn{2}{|c|}{ MCS-5 } & \multicolumn{2}{c|}{ MCS-6 } & \multicolumn{2}{c|}{ MCS-7 } \\
\hline Node & $\begin{array}{c}\text { PER } \\
(\mathbf{\%})\end{array}$ & $\begin{array}{c}\text { PSNR } \\
(\mathbf{d B})\end{array}$ & $\begin{array}{c}\text { PER } \\
(\mathbf{\%})\end{array}$ & $\begin{array}{c}\text { PSNR } \\
(\mathbf{d B})\end{array}$ & $\begin{array}{c}\text { PER } \\
(\mathbf{\%})\end{array}$ & $\begin{array}{l}\text { PSNR } \\
(\mathbf{d B})\end{array}$ \\
\hline \hline 1 & 0.00 & 47.17 & 0.00 & 47.17 & 0.13 & 41.76 \\
\hline 2 & 0.00 & 47.17 & 0.00 & 47.17 & 0.05 & 44.41 \\
\hline 3 & 0.00 & 47.05 & 0.00 & 47.17 & 0.06 & 43.93 \\
\hline 4 & 0.00 & 47.17 & 0.00 & 47.06 & 0.06 & 44.30 \\
\hline 5 & 0.00 & 47.17 & 0.00 & 47.17 & 0.14 & 41.96 \\
\hline 6 & 0.00 & 47.14 & 0.02 & 45.72 & 0.41 & 37.99 \\
\hline 7 & 0.01 & 46.62 & 0.02 & 45.58 & 0.27 & 39.76 \\
\hline 8 & 0.00 & 47.10 & 0.04 & 45.21 & 1.53 & 32.30 \\
\hline 9 & 0.01 & 46.84 & 0.03 & 45.96 & 0.26 & 39.15 \\
\hline 10 & 0.00 & 47.05 & 0.02 & 45.55 & 0.38 & 38.21 \\
\hline 11 & 0.00 & 47.17 & 0.22 & 39.69 & 0.60 & 36.20 \\
\hline 12 & 0.00 & 46.79 & 0.11 & 42.46 & 1.25 & 32.98 \\
\hline 13 & 0.01 & 46.29 & 0.09 & 43.25 & 5.23 & 27.61 \\
\hline 14 & 0.00 & 46.95 & 0.10 & 43.15 & 1.25 & 33.08 \\
\hline 15 & 0.00 & 47.08 & 0.22 & 40.24 & 0.57 & 36.24 \\
\hline 16 & 0.00 & 47.17 & 0.00 & 47.17 & 0.00 & 47.17 \\
\hline 17 & 0.00 & 46.84 & 0.07 & 43.83 & 1.45 & 32.52 \\
\hline 18 & 0.05 & 44.14 & 0.24 & 39.64 & 6.80 & 26.57 \\
\hline 19 & 0.00 & 47.09 & 0.07 & 43.15 & 1.40 & 32.68 \\
\hline 20 & 0.00 & 47.17 & 0.00 & 47.17 & 0.00 & 47.17 \\
\hline 21 & 0.05 & 44.36 & 0.29 & 39.20 & 4.42 & 28.14 \\
\hline 22 & 0.04 & 45.22 & 0.15 & 41.24 & 2.94 & 29.63 \\
\hline 23 & 0.03 & 45.30 & 0.09 & 43.26 & 1.66 & 31.99 \\
\hline 24 & 0.00 & 46.98 & 0.03 & 45.51 & 1.56 & 32.03 \\
\hline 25 & 0.03 & 44.83 & 0.58 & 36.00 & 6.39 & 26.77 \\
\hline 26 & 0.03 & 45.81 & 0.52 & 36.72 & 4.34 & 28.15 \\
\hline 27 & 0.03 & 45.12 & 0.42 & 37.68 & 2.26 & 30.70 \\
\hline 28 & 0.00 & 47.15 & 0.03 & 45.16 & 1.81 & 31.69 \\
\hline 29 & 0.02 & 45.99 & 0.16 & 40.57 & 3.20 & 29.33 \\
\hline 30 & 0.00 & 46.84 & 0.14 & 41.66 & 2.44 & 30.42 \\
\hline 31 & 0.00 & 47.04 & 0.02 & 46.03 & 0.80 & 35.12 \\
\hline 32 & 0.00 & 47.02 & 0.01 & 46.17 & 0.23 & 40.12 \\
\hline \hline
\end{tabular}

are located underneath one of the antennas placed at the edge of the coverage area. However, using the Infrastructures of Case A and Case B and MCS-6, 13 nodes, almost half of the multicast group in the sector considered, did not get an average PSNR of at least $36 \mathrm{~dB}$. Therefore, the distributed antennasbased infrastructure of Case $\mathrm{C}$ shown in Fig. 2 is highly beneficial even at a PHY data rate of $58.5 \mathrm{Mbps}$ with a code rate of $1 / 2$. Moreover some of these nodes i.e 10 nodes for Case $\mathrm{A}$ and 5 nodes for Case B obtained an average PSNR smaller than $30 \mathrm{~dB}$. Hence there was a considerable gain even for MCS-6 when Setup C was deployed.

For MCS-7, with the use of 8 transmit antennas as shown in Fig. 2, most of the multicast group members i.e. 25 nodes out of the 32 nodes studied, experienced an improvement in PER over the Infrastructure of case A i.e. an AP with one transmit antenna. However, more than half of the receivers (18 nodes) did not reach the desirable $36 \mathrm{~dB}$ average PSNR. But with the use of Infrastructure of Case A, 26 nodes out of the 32 nodes had a PSNR smaller than $36 \mathrm{~dB}$. But considering the overall performance, independent of which Infrastructure is deployed, one should either consider a smaller coverage area, or else combine the system of distributed antennas and packet repetition with a Forward Error Correction Scheme such as RS coding.

\section{DISTRIBUTED ANTENNAS-BASED INFRASTRUCTURE AND UNICAST}

The IEEE 802.11n standard exploits MIMO techniques to increase robustness, transmitted bit rate, or both [1]. The work presented in this paper shows that separating the antennas and distributing them around the periphery of the coverage area benefits multicasting, due to peculiarities of the transmission as specified by the standard. In our application it is required that the WLAN is used in normal unicast mode when it is not transmitting multicast packets. In order to allow this, we propose the use of two sets of antennas, switching between the two depending on whether multicast or unicast packets have to be transmitted.

Therefore when the AP has a unicast data packet it employs the antennas placed in the middle of the coverage area as shown in Fig. 5. But when it has a multicast or a broadcast packet it switches to the other antennas in order to employ the Distributed Antennas-Based Infrastructure as depicted in Fig. 6.

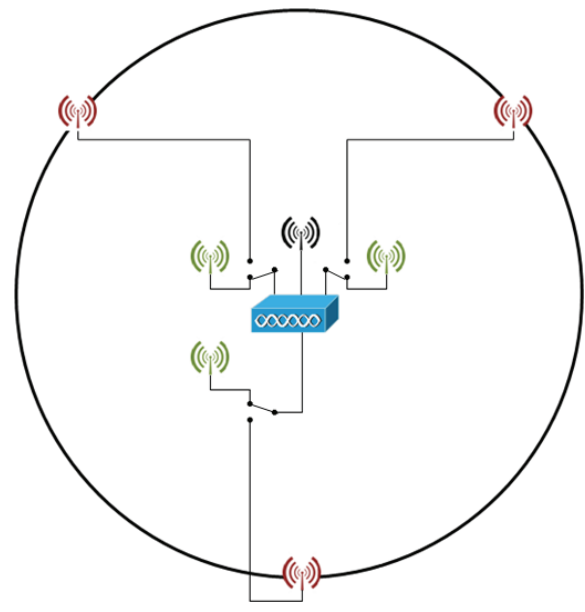

Fig. 5. Antenna Selection for Unicast Transmission

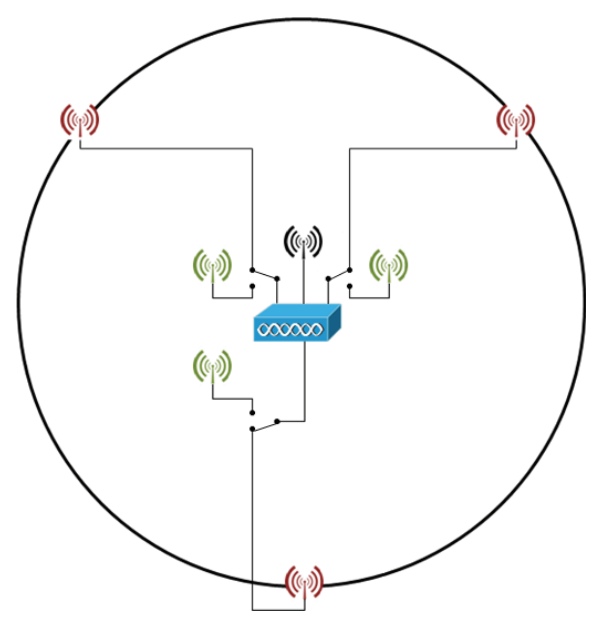

Fig. 6. Antenna Selection for Multicast Transmission 


\section{CONCLUSION}

Although the problems that wireless multicast over IEEE $802.11 \mathrm{n}$ faces are due to the lack of reliability at the Medium Access Control Layer, this paper shows that one can enhance the Infrastructure, in order to reduce the Packet Error Rate which will then affect the average PSNR obtained. The solution presented, which consists of two APs having four transmit antennas each, or else an AP with antenna selection, is simple to implement and practical. One can use amplified antenna feeders to achieve the proposed Infrastructure. On the other hand all IEEE $802.11 \mathrm{n}$ receivers can join the multicast group and benefit from a better channel quality, even at the least resilient modulation and coding schemes such as MCS-5, 6 and 7. Although this solution does not propose the use of data rate adaptation, it still solves the "Performance Anomaly Problem" because it builds an Infrastructure which can use faster Physical Layer data rates, instead of depending on the most robust one. Another important point, besides the simplicity in the implementation of the Infrastructure, is the fact that the code rate is that of $1 / 2$ and the delay introduced is that of 1 time slot. This is a considerable improvement on cooperative schemes, in which each relay has to retransmit the same data packet reducing the code rate. In previously proposed cooperative schemes which have been shown to be beneficial to multicast over wireless, at least two relays are required to retransmit the data from the Access Point to the multicast group members which can be located in any part of the coverage area. Therefore, previous cooperative schemes achieved at maximum a code rate of $1 / 3$ while the cooperative system presented in this paper achieves the required low PER with a code rate of $1 / 2$.

\section{ACKNOWLEDGMENT}

The authors would like to acknowledge IST project IST2000-30148 I-METRA for the Matlab Code which simulates the IEEE $802.11 \mathrm{n}$ channels

\section{REFERENCES}

[1] Wireless LAN Medium Access Control (MAC) and Physical Layer (PHY) Specifications, Amendment 5: Enhancements for Higher Throughput, IEEE Std. 802.11n ${ }^{\mathrm{TM}}, 2009$.

[2] Wireless LAN Medium Access Control (MAC) and Physical Layer (PHY) Specifications - Amendment 2: MAC Enhancements for robust audio video streaming, IEEE Std. 802.11 aa, 2012.

[3] A. Ashley and N. Kakani, "Splitting GCR from DMS," Tech. Rep. IEEE 802.11-10/1445r2, Jan. 2011.
[4] Z. Feng, G. Wen, C. Yin and H. Liu, "Video stream groupcast optimization in WLAN," in Proc. International Conference on Internet Technology and Applications 2010, Aug. 2010, pp. 1-4.

[5] A. Ashley and S. Pandey, "Normative text for LB173 GCR comment resolutions," Tech. Rep. IEEE 802.11/0300r1, Mar. 2011.

[6] A. Kamerman and L. Monteban, "WaveLAN-II: a highperformance wireless LAN for the unlicensed band," Bell Labs Technical Journal, vol. 2, pp. 118-13, 1997.

[7] M. Heusse, F. Rousseau, G. Berger-Sabbatel, and A. Duda, "Performance anomaly of 802.11b," in Proc. INFOCOM'03, Apr. 2003, pp. 836-843.

[8] D. Dujovne and T. Turletti, "Multicast in 802.11 WLANs: an experimental study," in Proc. MSWiM'06, 2006, pp. 130-138.

[9] J. M. Vella and S. Zammit, "A survey of multicasting over wireless access networks," IEEE Commun. Surveys Tuts., in press.

[10] J.-M. Vella and S. Zammit, "Infrastructure-dependent wireless multicast over 802.11n WLAN," in Proc. SoftCOM'11, Sept. 2011, pp $1-5$.

[11] J.-M. Vella and S. Zammit, "Packet losses of multicast over 802.11n heterogeneous wireless local area network," in Proc. IWSSIP'12, Apr. 2012, pp. 300-303.

[12] J.-M. Vella and S. Zammit, "An analytical study of infrastructuredependent wireless multicast over IEEE 802.11n WLANs," IEEE EUROCON 2013, submitted for publication.

[13] "QHS600 Reference Design" [Online]. Available: http://www.quantenna.com/pdf/QHS600.pdf

[14] Y. Zhou, Z. Pan, L. Tian, G. Sun and J. Shi, "Cell throughput of multicast services in OFDM-based distributed antenna systems, " in Proc. VTC'11, May 2011, pp. 1-5

[15] A. Miu, G. Tan, H. Balakrishnan and J. Apostolopoulos, "Divert: fine-grained path selection for wireless LANs," in Proc. MobiSys'04, Jun. 2004, pp. 203-216.

[16] J.-M. Vella and S. Zammit, "Infrastructure dependent wireless multicast - the effect of spatial diversity and error correction," in Proc. CoCoS'12, Oct. 2012, pp. 989-996.

[17] J. P. Kermoal, L. Schumacher, K. Pedersen, P. Mogensen and F. Frederiksen, "A stochastic MIMO radio channel model with experimental validation," IEEE J. Sel. Areas Commun., vol. 20, no. 6, pp. 1211-1226, Aug 2002.

[18] E. Perahia and R. Stacey, "Next Generation Wireless LANs Throughput, Robustness, and Reliability in 802.11n", Cambridge University Press, 2008, pp. 144-147.

[19] V. Erceg et al. "TGn channel models," IEEE 802.11-03/940r04.

[20] B. Bjerke, J. Ketchum, R. Walton, S. Nanda, I. Medvedev, M. Wallace, and S. Howard, "Packet error probability prediction for system level simulations of MIMO-OFDM based 802.11n WLANs," in Proc. ICC'05, May 2005, pp. 2538-2542.

[21] Q. Xia, M. Hamdi , and K. B. Letaief, "Open-loop link adaptation for next generation IEEE 802.11 n wireless networks, "IEEE Trans. Veh. Technol., vol. 58, no.7, pp. 3713-3725, Sept.2009.

[22] "The ns-3 Network Simulator," [Online], Available: http://www.nsnam.org/.

[23] "Football Sequence," [Online], Available: http://media.xiph.org/video/derf/.

[24] "ffmpeg-0.11," [Online]. Availabel: http://ffmpeg.org/.

[25] S. Zammit, "Digital video transmission over wireless networks," Ph.D. dissertation, Aston University, 1996. 